\title{
Strong surjectivity of mappings of some 3-complexes into 3-manifolds
}

\author{
by \\ Claudemir Aniz (Campo Grande)
}

\begin{abstract}
Let $K$ be a $C W$-complex of dimension 3 such that $H^{3}(K ; \mathbb{Z})=0$, and $M$ a closed manifold of dimension 3 with a base point $a \in M$. We study the problem of existence of a map $f: K \rightarrow M$ which is strongly surjective, i.e. such that $\operatorname{MR}[f, a] \neq 0$. In particular if $M=S^{1} \times S^{2}$ we show that there is no $f: K \rightarrow S^{1} \times S^{2}$ which is strongly surjective. On the other hand, for $M$ the non-orientable $S^{1}$-bundle over $S^{2}$ there exists a complex $K$ and $f: K \rightarrow M$ such that $\operatorname{MR}[f, a] \neq 0$.
\end{abstract}

1. Introduction. Given a map $f: K \rightarrow M$ between topological spaces, and $a \in M$ an arbitrary point, recall that

$$
\operatorname{MR}[f, a]=\min \left\{\#\left(g^{-1}(a)\right) \mid g \in[f]\right\},
$$

where [ ] means homotopy class. We say that a map $f: K \rightarrow M$ is strongly surjective if any map homotopic to it is surjective (or, equivalently, if $\operatorname{MR}[f, a] \neq 0$ for some $a \in M)$. One of the main goals of root theory is to compute $\operatorname{MR}[f, a]$, which for a connected manifold $M$ is independent of $a$. The Nielsen root theory gives us the Nielsen root number, denoted by $N(f, a)$, which is a lower bound for $\operatorname{MR}[f, a]$ (see [TK, Chapter 5]). When $K$ and $M$ are compact triangulable manifolds of the same dimension $r \geq 3$, we have $N(f, a)=\operatorname{MR}[f, a]$ (see [BR3, Proposition 4]). In [BR1], making use of obstruction theory, it was proven that if $f: K \rightarrow M$ is a map from a finite simplicial complex of dimension $r \geq 3$ to a manifold such that $\pi_{p}(M, M-a)=0$ for all $0<p<r$, then it is possible to find a map $g \in[f]$ having exactly $N(f, a)$ roots. None of the results above guarantees that there is a map $f: K \rightarrow M$ such that $\operatorname{MR}[f, a] \neq 0$. Further, if the complex $K$ satisfies $H^{3}(K ; \mathbb{Z})=0$, the Nielsen root number (given in terms of a local index of Nielsen root classes) is always zero.

2000 Mathematics Subject Classification: Primary 55M20; Secondary 55S35, 55N25.

Key words and phrases: strongly surjective map, cohomology with local coefficients, complexes with zero top cohomology, manifolds with abelian fundamental group. 
Root theory may be considered as a particular case of coincidence theory (see [BR3]). Recent works have studied coincidence theory for pairs of maps $(f, g): K \rightarrow M$, having as domain a simplicial complex $K$, and as codomain a manifold $M$, both of the same dimension. In $[\mathrm{G}]$, using obstruction theory, the index of a coincidence Nielsen class of a pair $(f, g)$ is defined. In [BG1] and [BG2] Nielsen type numbers are defined for coincidences so that they are lower bounds and, under certain hypotheses, agree with $\mathrm{MC}[f, g]$, i.e. the minimal number of coincidence points among all maps $f^{\prime}, g^{\prime}$ homotopic to $f, g$, respectively. So the same type of questions studied here can be asked in coincidence theory.

In this work we assume $K$ is a $C W$-complex and $M$ a closed manifold, both of the same dimension $r=3$. The main results are (see Theorems 5.7, $5.8,5.9,5.11,5.14$ and 5.16):

1. There is no map $f: K \rightarrow M$ which is strongly surjective if $K$ is a 3 -complex with $H^{3}(K ; \mathbb{Z})=0$ and $M$ is either $S^{1} \times S^{2}, S^{1} \times S^{1} \times S^{1}$, or a lens space.

2. There is no map $f: K \rightarrow M$ which is strongly surjective if $K$ is a 3complex and $H^{3}(K ; \widetilde{\mathbb{Z}})=0$ and $M$ is either $S^{1} \times \mathbb{P}^{2}\left(\mathbb{P}^{n}\right.$ denotes the $n$ dimensional real projective space) or $M_{A}=S^{2} \times[0,1] /(x, 0) \sim(-x, 1)$. Here $H^{3}(K ; \widetilde{\mathbb{Z}})$ is the cohomology with an arbitrary local coefficient system $\widetilde{\mathbb{Z}}$.

3. There exists a strongly surjective map $K \rightarrow M_{A}$, where $M_{A}$ is the 3 -manifold defined in item 2 above and $K$ is a certain 3 -complex with $H^{3}(K ; \mathbb{Z})=0$.

The organization of this paper is as follows. In Section 2 we present some results on group rings. In Section 3 we compute cohomology groups with local coefficients for certain $C W$-complexes. These complexes will have the property that $H^{3}(K ; \mathbb{Z})=0$, but the cohomology $H^{3}(K ; \widetilde{\mathbb{Z}})$ with local coefficients is non-trivial. Section 4 contains examples of complexes $K$ constructed from a given 2-dimensional complex and such that $H^{3}(K ; \mathbb{Z})=0$. In Section 5 the vanishing of the obstruction to deform a map $f: K \rightarrow M$ to a root free map is described in terms of solutions of a linear system $P x=k$ over $\mathbb{Z}\left[\pi_{1}(M)\right]$ (see Corollary 5.5).

This work is based on my doctoral thesis, written under the supervision of Professor Daciberg Lima Gonçalves.

2. Group ring. In this section we will present some results on group rings that will be used in Section 5. For details see [A].

The symbol $\mathbb{Z}[G]$ will mean the group ring of $G$ over the integers $\mathbb{Z}$. The map $\varepsilon: \mathbb{Z}[G] \rightarrow \mathbb{Z}$ given by $\sum_{i=1}^{p} n_{i} g_{i} \rightarrow \sum_{i=1}^{p} n_{i}$ is a ring homomorphism 
called the augmentation map. Its kernel $\Delta_{\mathbb{Z}}(G)=\operatorname{Ker} \varepsilon$ is the augmentation ideal of $\mathbb{Z}[G]$. The ring $\mathbb{Z}[G]$ is commutative if the group $G$ is abelian.

If $G=\bigoplus_{i=1}^{p} \mathbb{Z}$, then $\mathbb{Z}\left[\bigoplus_{i=1}^{p} \mathbb{Z}\right]=\mathbb{Z}\left[x_{1}, \ldots, x_{p}, x_{1}^{-1}, \ldots, x_{p}^{-1}\right]$ is the ring of Laurent polynomials in $p$ variables. If $G=\mathbb{Z}_{p}$ is the cyclic group of order $p, p \geq 2$, then $\mathbb{Z}\left[\mathbb{Z}_{p}\right]=\mathbb{Z}[x] /\left(x^{p}-1\right)$.

The results that follow will be used in Section 5 .

LEMMA 2.1. The ideal $\Delta_{\mathbb{Z}}\left(\bigoplus_{i=1}^{p} \mathbb{Z}\right)$ is generated by $x_{i}-1$ for $i=1, \ldots, p$. The ideal $\Delta_{\mathbb{Z}}\left(\mathbb{Z}_{p}\right)$ is generated by $x-1$.

LEMMA 2.2. The kernel of the homomorphism $\bar{\varepsilon}: \mathbb{Z}\left[x, x^{-1}\right] \rightarrow \mathbb{Z}$ given by

$$
\bar{\varepsilon}\left(x^{j}\right)= \begin{cases}-1 & \text { if } j \text { is odd }, \\ 1 & \text { if } j \text { is even },\end{cases}
$$

is the ideal generated by the element $x+1$.

LEMMA 2.3. Let $A$ be an $m \times n$ matrix over $\mathbb{Z}\left[\bigoplus_{i=1}^{p} \mathbb{Z}\right]$ with $m \leq n$ such that all $m \times m$ minors of the matrix $\varepsilon(A)=\left[\varepsilon\left(a_{i j}\right)\right]$ are relatively prime. The system $A x=k$ has a solution over $\mathbb{Z}\left[\bigoplus_{i=1}^{p} \mathbb{Z}\right]$ if, and only if, the systems $A x=k\left(x_{i}-1\right)$ have solutions over $\mathbb{Z}\left[\bigoplus_{i=1}^{p} \mathbb{Z}\right]$ for $i=1, \ldots, p$.

Proof. Let $\left\{d_{1}, \ldots, d_{r}\right\}$ be the set of all $m \times m$ minors of $A$. Since $\varepsilon\left(d_{1}\right), \ldots, \varepsilon\left(d_{r}\right)$ are relatively prime, there are integers $p_{1}, \ldots, p_{r}$ so that $p_{1} d_{1}+\cdots+p_{r} d_{r} \in \mathbb{Z}\left[\bigoplus_{i=1}^{p} \mathbb{Z}\right]$ has augmentation 1 . From Lemma 2.1, we can find $q_{1}, \ldots, q_{p} \in \mathbb{Z}\left[\bigoplus_{i=1}^{p} \mathbb{Z}\right]$ with

$$
p_{1} d_{1}+\cdots+p_{r} d_{r}=q_{1}\left(x_{1}-1\right)+\cdots+q_{p}\left(x_{p}-1\right)+1 .
$$

But this means that the set $\left\{d_{1}, \ldots, d_{r}, x_{1}-1, \ldots, x_{p}-1\right\}$ is not contained in any maximal ideal $\mathfrak{m}$. Since the systems $A x=k d_{i}$ have solutions in $\mathbb{Z}\left[\bigoplus_{i=1}^{p} \mathbb{Z}\right]$ for $i=1, \ldots, r$, the existence a solution for the systems $A x=$ $k\left(x_{i}-1\right), i=1, \ldots, p$, implies that the system $A x=k$ also has a solution ([HG, Proposition 1]).

LEMMA 2.4. If $G=\mathbb{Z} \times \mathbb{Z}_{2}$, the kernel of the homomorphism $\widetilde{\varepsilon}: \mathbb{Z}\left[\mathbb{Z} \times \mathbb{Z}_{2}\right]$ $\rightarrow \mathbb{Z}$ given by $\widetilde{\varepsilon}\left(y^{k} x\right)=-1$ and $\widetilde{\varepsilon}\left(y^{k}\right)=1$ is generated by $x+1$ and $y-1$.

3. Cohomology with local coefficients. In this section we will look at cohomology with local coefficients. The basic references are [WG] and $[\mathrm{A}]$.

For $B$ a path connected topological space, a local coefficient system over $B$ is determined by a group homomorphism

$$
\Omega: \pi_{1}\left(B, b_{0}\right) \rightarrow \operatorname{Aut}(G),
$$

where $b_{0} \in B$ and $\operatorname{Aut}(G)$ is the group of automorphisms of an abelian group $G$. The following coefficient systems will be used in this work. The bijection $\Omega(\theta): \pi_{1}\left(B, b_{0}\right) \rightarrow \pi_{1}\left(B, b_{0}\right)$ given by $\Omega(\theta)(\alpha)=\theta \alpha$ for $\theta, \alpha \in \pi_{1}\left(B, b_{0}\right)$, 
extends to an isomorphism of abelian groups

$$
\Omega(\theta)_{\mathbb{Z}}: \mathbb{Z}\left[\pi_{1}\left(B, b_{0}\right)\right] \rightarrow \mathbb{Z}\left[\pi_{1}\left(B, b_{0}\right)\right] .
$$

The homomorphism $\Omega: \pi_{1}\left(B, b_{0}\right) \rightarrow \operatorname{Aut}\left(\mathbb{Z}\left[\pi_{1}\left(B, b_{0}\right)\right]\right)$ given by $\Omega(\theta)=$ $\Omega(\theta)_{\mathbb{Z}}$ is a local coefficient system. Similarly for

$$
\Omega(\theta): \pi_{1}\left(B, b_{0}\right) \rightarrow \pi_{1}\left(B, b_{0}\right)
$$

given by $\Omega(\theta)(\alpha)=\theta \alpha \theta^{-1}$.

Let $K$ be a connected $C W$-complex and $p: \widetilde{K} \rightarrow K$ its universal covering. Let $C(\widetilde{K})$ be the cellular chain complex $\left\{C_{n}(\widetilde{K}), \widetilde{\partial}_{n}\right\}$ of $\widetilde{K}$. The group $\Pi=\pi_{1}\left(K, k_{0}\right)$ acts on $C_{n}(\widetilde{K})$, and consequently $C_{n}(\widetilde{K})$ becomes a left $\mathbb{Z}[\Pi]$-module. If $\Omega$ is a local coefficient system on $K$, the group $\Pi$ acts on $G$, and consequently $G$ becomes a left $\mathbb{Z}[\Pi]$-module. Let $\operatorname{Hom}^{\Pi}\left(C_{n}(\widetilde{K}) ; G\right)$ be the group of all $\mathbb{Z}[\Pi]$-homomorphisms. Write

$$
\widetilde{\delta}_{n}: \operatorname{Hom}^{\Pi}\left(C_{n-1}(\widetilde{K}) ; G\right) \rightarrow \operatorname{Hom}^{\Pi}\left(C_{n}(\widetilde{K}) ; G\right)
$$

for the homomorphism defined by $\widetilde{\delta}_{n}(\alpha)=\alpha \circ \widetilde{\partial}_{n}$. Thus we have a cochain complex

$$
\left\{\operatorname{Hom}^{\Pi}\left(C_{n}(\widetilde{K}) ; G\right), \widetilde{\delta}^{n}\right\}
$$

and

$$
H^{n}(K ; \Omega)=\operatorname{Ker} \widetilde{\delta}_{n+1} / \operatorname{Im} \widetilde{\delta}_{n}
$$

is the cohomology group of $K$ with local coefficients $\Omega$.

REMARK 3.1. If $K$ is finite of dimension $r$, then $C_{n}(\widetilde{K})=0$ for $n>r$, and

$$
H^{r}(K ; \Omega)=\operatorname{Hom}^{\Pi}\left(C_{r}(\widetilde{K}) ; G\right) / \operatorname{Im} \widetilde{\delta}_{r} .
$$

We will study the case $G=\mathbb{Z}$ in detail. To obtain the cohomology with trivial coefficients $H^{n}(K ; \mathbb{Z})$, we need only consider the simple coefficient system $\Omega: \Pi \rightarrow \operatorname{Aut}(\mathbb{Z})$ where $\Omega(\alpha)=$ identity for all $\alpha \in \Pi$.

DEFINITION 3.2. The symbol $\widetilde{\mathbb{Z}}$ will represent any local coefficient system $\Omega: \Pi \rightarrow \operatorname{Aut}(\mathbb{Z})$ on $K$ with $\Omega$ surjective.

Suppose that $K$ is a finite $C W$-complex of dimension $r \geq 1$, with $n$ cells of dimension $r-1$ and $m$ cells of dimension $r$.

Lemma 3.3. The group $H^{r}(K ; \mathbb{Z})$ is finite of odd cardinality if, and only if , the group $H^{r}(K ; \widetilde{\mathbb{Z}})$ is finite of odd cardinality.

Proof. The boundary operator $\widetilde{\partial}_{r}: H_{r}\left(\widetilde{K}^{r}, \widetilde{K}^{r-1}\right) \rightarrow H_{r-1}\left(\widetilde{K}^{r-1}, \widetilde{K}^{r-2}\right)$ is given by the matrix

$$
A=\left[\begin{array}{ccc}
\widetilde{a}_{11} & \ldots & \widetilde{a}_{1 m} \\
\ldots & \ldots & \ldots \\
\widetilde{a}_{n 1} & \ldots & \widetilde{a}_{n m}
\end{array}\right]
$$


with columns defined by

$$
\widetilde{\partial}_{r}\left(\widetilde{e}_{i}^{r}\right)=\widetilde{a}_{1 i} \widetilde{e}_{1}^{r-1}+\cdots+\widetilde{a}_{n i} \widetilde{e}_{n}^{r-1}
$$

for $i=1, \ldots, m$. Consider $\phi \in \operatorname{Hom}^{\Pi}\left(C_{r-1}(\widetilde{K}) ; \mathbb{Z}\right)$ given by $\phi\left(\widetilde{e}_{j}^{r-1}\right)=k_{j}$ for $j=1, \ldots, n$. The homomorphism $\widetilde{\delta}_{r}(\phi) \in \operatorname{Hom}^{\Pi}\left(C_{r}(\widetilde{K}) ; \mathbb{Z}\right)$ has the property that $\widetilde{\delta}_{r}(\phi)\left(\widetilde{e}_{i}^{r}\right)=\phi \circ \widetilde{\partial}_{r}\left(\widetilde{e}_{i}^{r}\right)=\phi\left(\widetilde{a}_{1 i} \widetilde{e}_{1}^{r-1}+\cdots+\widetilde{a}_{n i} \widetilde{e}_{n}^{r-1}\right)=\widetilde{a}_{i 1} \phi\left(\widetilde{e}_{1}^{r-1}\right)+$ $\cdots+\widetilde{a}_{n i} \phi\left(\widetilde{e}_{n}^{r-1}\right)=\widetilde{a}_{1 i} k_{1}+\cdots+\widetilde{a}_{n i} k_{n}$. Notice that each $\widetilde{a}_{i j} \in \mathbb{Z}[\Pi]$ is of the form $\sum_{k=1}^{p} n_{k} \alpha_{k}$, and the action is given by $\left(\sum_{k=1}^{p} n_{k} \alpha_{k}\right) 1=\bar{\varepsilon}\left(\widetilde{a}_{i j}\right)=$ $\sum_{k=1}^{p} n_{k}\left(\alpha_{k 1}\right)$, where $\left(\alpha_{k 1}\right)$ is either 1 or -1 ,

$$
\left[\begin{array}{ccc}
\bar{\varepsilon}\left(\widetilde{a}_{11}\right) & \ldots & \bar{\varepsilon}\left(\widetilde{a}_{n 1}\right) \\
\ldots & \ldots & \ldots \\
\bar{\varepsilon}\left(\widetilde{a}_{1 m}\right) & \ldots & \bar{\varepsilon}\left(\widetilde{a}_{n m}\right)
\end{array}\right]\left[\begin{array}{c}
k_{1} \\
\vdots \\
k_{n}
\end{array}\right]=\left[\begin{array}{c}
\phi \circ \widetilde{\partial}_{r}\left(\widetilde{e}_{1}^{r}\right) \\
\vdots \\
\phi \circ \widetilde{\partial}_{r}\left(\widetilde{e}_{m}^{r}\right)
\end{array}\right] .
$$

This means that the diagram

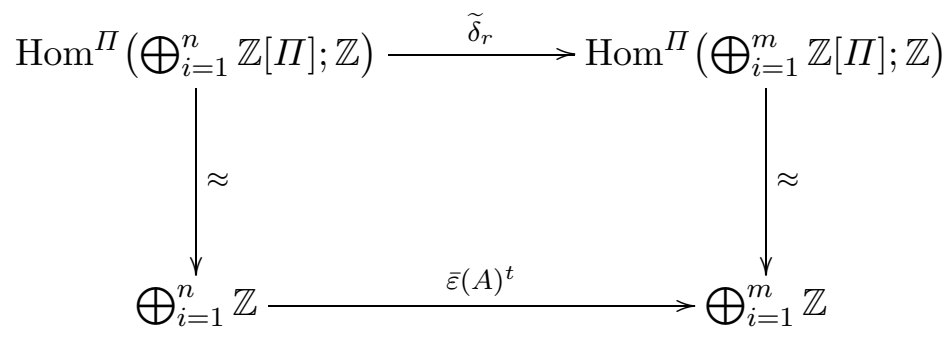

is commutative. Therefore

$$
H^{r}(K ; \widetilde{\mathbb{Z}})=\left(\bigoplus_{i=1}^{m} \mathbb{Z}\right) / \operatorname{Im}\left(\bar{\varepsilon}(A)^{t}\right)
$$

By [BR2, Chapter 3, Proposition 15], the cardinality of $H^{r}(K ; \mathbb{Z})$ is $\operatorname{gcd}\left\{\varepsilon\left(d_{1}\right), \ldots, \varepsilon\left(d_{r}\right)\right\}$, where $\varepsilon\left(d_{i}\right)$ are the minors of $\varepsilon(A)^{t}$. If this number is odd, at least one of the minors is odd. As $\varepsilon\left(\widetilde{a}_{i j}\right) \equiv \bar{\varepsilon}\left(\widetilde{a}_{i j}\right) \bmod 2, \bar{\varepsilon}(A)^{t}$ will also have minors of odd order (see $[\mathrm{A}]$ ). Again by [BR2, Chapter 3, Proposition 15$], H^{r}(K ; \widetilde{\mathbb{Z}})$ has finite and odd cardinality.

Next we give an example of a 3 -complex with $H^{3}(K ; \mathbb{Z})=0$ and $H^{3}(K ; \Omega) \neq 0$.

Consider the 2-complex $K^{2}=S^{1} \vee S^{2}$, the bouquet of a circle and a sphere. Its universal cover $\widetilde{K}^{2}$ is the straight line with a 2 -sphere attached to each integer. Since $\pi_{2}\left(K^{2}\right)=\pi_{2}\left(\widetilde{K}^{2}\right)=H_{2}\left(\widetilde{K}^{2}\right)$ and $\pi_{2}\left(K^{2}, x_{0}\right)=\mathbb{Z}\left[t, t^{-1}\right]$, all maps $f:\left(S^{2}, s_{0}\right) \rightarrow\left(S^{1} \vee S^{2}, x_{0}\right)$ are of the form $f=\sum \alpha_{i} t^{i}$. Let $K$ be the complex obtained from $K^{2}$ by attaching one 3 -cell via $2 t-1$. The boundary operator

$$
0 \rightarrow H_{3}\left(\widetilde{K}^{3}, \widetilde{K}^{2}\right) \stackrel{\widetilde{\partial}_{3}}{\longrightarrow} H_{2}\left(\widetilde{K}^{2}, \widetilde{K}^{1}\right)
$$


is given by $\widetilde{\partial}_{3}(y)=(2 t-1) y$, and from the proof of the previous lemma, $H^{3}(K ; \mathbb{Z})=0$.

Consider the local coefficient system $\Omega: \pi_{1}(K)=\mathbb{Z} \rightarrow \operatorname{Aut}\left(\mathbb{Z}\left[t, t^{-1}\right]\right)$ on $K$, given by

$$
\Omega(t)\left(\sum_{i=1}^{p} n_{i} t^{k_{i}}\right)=\sum_{i=1}^{p} n_{i} t^{1+k_{i}} .
$$

The next result is proved in $[\mathrm{A}]$.

Proposition 3.4. $H^{3}(K ; \Omega)$ is isomorphic to the subgroup of $\mathbb{Q}$ generated by the elements whose denominators are powers of 2 .

REMARK 3.5. Observe that in Lemma 3.3, if we have $H^{r}(K ; \mathbb{Z})$ finite, we cannot guarantee that $H^{r}(K ; \widetilde{\mathbb{Z}})$ is finite. Consider the following example: let $K$ be the 3 -complex obtained from $K^{2}=S^{1} \vee S^{2}$ by attaching a 3-cell by the map $t+1$. Consider the system $\widetilde{\mathbb{Z}}$ given by the action $t 1=-1$, where $t \in \pi_{1}(K)=\mathbb{Z}$ is the generator. It follows from the proof of Lemma 3.3 that $H^{3}(K ; \mathbb{Z})=\mathbb{Z}_{2}$ and $H^{3}(K ; \widetilde{\mathbb{Z}})=\mathbb{Z}$.

REMARK 3.6. The hypothesis that $H^{r}(K ; \mathbb{Z})=0$ and $H^{r}(K ; \widetilde{\mathbb{Z}})=0$ does not guarantee that $H^{r}(K ; \Omega)=0$. The complex $K$ obtained from $K^{2}=S^{1} \vee S^{2}$ by attaching a 3 -cell via the map $2 t^{2}-1$ satisfies $H^{3}(K ; \mathbb{Z})=0$, $H^{3}(K ; \widetilde{\mathbb{Z}})=0$, where $\widetilde{\mathbb{Z}}$ is the system described in the previous remark, and $H^{3}(K ; \Omega) \neq 0$.

Let $K$ be a finite connected $C W$-complex of dimension $r \geq 3, M$ a closed connected manifold of the same dimension as $K$ that admits a structure of $C W$-complex, and $M^{r-1}$ the $(r-1)$-skeleton of $M$. The obstruction to deforming a map $f: K \rightarrow M$ into $M^{r-1}$ is denoted by $\omega^{r}(f)$ and lies in $H^{r}\left(K ; f^{*} \pi_{r}\left(M, M^{r-1}\right)\right)$.

THEOREM 3.7. Let $f: K \rightarrow M$ be a map such that $f\left(K^{r-1}\right) \subset M^{r-1}$ and $\omega^{r}(f)=0$. Then there exists a homotopy $\left\{f_{t}\right\}$ of $f$ such that $f_{1}(K) \subset M^{r-1}$. In fact, the homotopy can be chosen to be constant on $K^{r-2}$.

The first part of Theorem 3.7 follows from [WG, Chapter VI, Section 6, Theorem 6.3]. For the second part, i.e. that the homotopy can be taken relative to $K^{r-2}$, we can argue as follows: Because the pair $\left(M, M^{r-1}\right)$ is $(r-1)$-connected, we can assume that the homotopy between $f$ and $f_{1}$ maps $K^{r-2} \times I$ into $M^{r-1}$. Now by the AHEP (absolute homotopy extension property; see [H, Chapter VI, Exercise E, p. 197]) the result follows.

REMARK 3.8. For any two points $x, y \in M$ there exists a homeomorphism $h: M \rightarrow M$ such that $h(x)=y$ and $h$ is homotopic to the identity map of $M$. This fact implies that the number $\operatorname{MR}[f, a]$ does not depend on the point $a$. If $M$ admits a $C W$-decomposition with only one cell $e_{M}$ 
of dimension $r$, then for the purpose of calculation of $\operatorname{MR}[f, a]$, we may assume that the point $a$ is in the interior of $e_{M}$. Since $M^{r-1}$ is a strong deformation retract of $M-\{a\}$, this means that $\operatorname{MR}[f, a]=0$ is equivalent to the possibility of deforming $f$ into $M^{r-1}$. Since $f$ is homotopic to a cellular map $f_{1}: K \rightarrow M$ we may assume that $f\left(K^{r-1}\right) \subset M^{r-1}$. By Theorem 3.7, $f$ can be deformed into $M^{r-1}$ if, and only if, the obstruction class $\omega^{r}(f) \in H^{r}\left(K ; f^{*} \pi_{r}\left(M, M^{r-1}\right)\right)$ is zero.

4. Complexes with zero top cohomology. For $K$ an $r$-dimensional $C W$-complex with $m$ cells of dimension $r$, and $n$ cells of dimension $r-1$, we have

$$
H^{r}(K ; \mathbb{Z})=\left(\bigoplus_{i=1}^{m} \mathbb{Z}\right) / \operatorname{Im}\left(\varepsilon(A)^{t}\right)
$$

If $n<m$, this quotient is infinite (see [BR2, Chapter 3, Proposition 15]). Hence, in order to have $H^{r}(K ; \mathbb{Z})$ finite we must have $m \leq n$. In particular this is the case when $H^{r}(K ; \mathbb{Z})=0$, as $m \leq n$ is a necessary condition to construct such complexes. In this case, $H^{r}(K ; \mathbb{Z})=0$ if, and only if, the $m \times m$ minors of the matrix $\varepsilon(A)^{t}$ are relatively prime.

Definition 4.1. When $m=n$, we will say that $K$ is square.

Lemma 4.2. If a complex $K^{r-1}$ satisfies either $H_{r-1}\left(K^{r-1}\right)=0$ or $\pi_{r-1}\left(K^{r-1}\right)=0$, then it is not possible to construct a complex $K=K^{r}$, from $K^{r-1}$, with $H^{r}(K ; \mathbb{Z})=0$.

Proof. The universal covering $p: \widetilde{K} \rightarrow K$ is a cellular map and hence it induces a homomorphism

$$
p_{*}: H_{r}\left(\widetilde{K}^{r}, \widetilde{K}^{r-1}\right)=\bigoplus_{i=1}^{m} \mathbb{Z}[\Pi] \rightarrow H_{r}\left(K^{r}, K^{r-1}\right)=\bigoplus_{i=1}^{m} \mathbb{Z}
$$

which is the augmentation on each summand. The commutative diagram

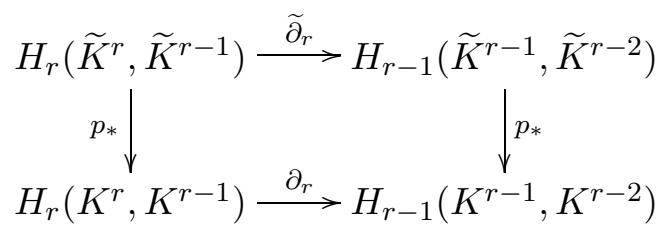

says that, if $\partial_{r}\left(e_{i}^{r}\right)=\left(a_{1 i}, \ldots, a_{n i}\right)$, then $\widetilde{\partial}_{r}\left(\widetilde{e}_{i}^{r}\right)=\left(\widetilde{a}_{1 i}, \ldots, \widetilde{a}_{n i}\right)$, where $\varepsilon\left(\widetilde{a}_{j i}\right)=a_{j i}$ for $j=1, \ldots, n$ and $i=1, \ldots, m$. A complex $K^{r}$ is obtained from $K^{r-1}$ by attaching $r$-cells via maps $\varphi: S^{r-1} \rightarrow K^{r-1}$, and if either $H_{r-1}\left(K^{r-1}\right)=0$ or $\pi_{r-1}\left(K^{r-1}\right)=0$, the induced homomorphism $\varphi_{*}: H_{r-1}\left(S^{r-1}\right) \rightarrow H_{r-1}\left(K^{r-1}\right)$ is zero. Since $\partial_{r}$ is defined in terms of $\varphi_{*}$, we have $\partial_{r}=0$. Therefore the matrix $\varepsilon(A)^{t}$ is zero. 
In particular $K^{r-1}$ cannot be a non-orientable closed manifold. In case $r=3, K^{2}$ is $S^{2}$, and consequently $K^{3}$ is simply connected.

COROLlary 4.3. The only 2-complex $K^{2}$ having a cell in each dimension $0,1,2$ and from which it is possible to construct a 3 -complex with $H^{3}(K ; \mathbb{Z})$ $=0$ is $S^{1} \vee S^{2}$.

Proof. Observe that $K^{2}$ is obtained from $S^{1}$ by attaching a 2-cell via a map $\varphi: S^{1} \rightarrow S^{1}$ of degree $n$. We will calculate $H_{2}\left(K^{2}\right)$. We have

$$
0 \rightarrow H_{2}\left(K^{2}, K^{1}\right) \stackrel{\partial_{2}}{\longrightarrow} H_{1}\left(K^{1}, K^{0}\right) \stackrel{\partial_{1}}{\longrightarrow} H_{0}\left(K^{0}\right) \rightarrow 0 .
$$

Here $\partial_{2}(p)=n p$ and $\partial_{1}=0$. Since $H_{2}\left(K^{2}\right)=\operatorname{Ker} \partial_{2}$, we have $H_{2}\left(K^{2}\right)=0$, when $n \neq 0$. From the previous lemma it is not possible to construct $K=K^{3}$ from such a $K^{2}$ with $H^{3}(K ; \mathbb{Z})=0$.

Next we will see some examples of 2-complexes from which it is possible to construct 3-complexes with trivial third cohomology group.

EXAMPLE 4.4. Let $K^{2}=S^{1} \vee \bigvee_{i=1}^{n} S^{2}$. Its universal cover $\widetilde{K}^{2}$ is the straight line together with a copy of $\bigvee_{i=1}^{n} S^{2}$ attached to each integral coordinate. Then $\pi_{2}\left(K^{2}\right)=H_{2}\left(\widetilde{K}^{2}\right)=\bigoplus_{i=1}^{n} \mathbb{Z}\left[t, t^{-1}\right]$, and each element is given by an $n$-tuple $\left(p_{1}(t), \ldots, p_{n}(t)\right)$. To get from $K^{2}$ a complex $K$ with $H^{3}(K ; \mathbb{Z})=0$ it is enough to attach $m 3$-cells, $m \leq n$, such that $\widetilde{\partial}_{3}\left(\widetilde{e}_{i}^{3}\right)=p_{1 i}(t) \widetilde{e}_{1}^{2}+\cdots+p_{n i}(t) \widetilde{e}_{n}^{2}$, where the $m \times m$ minors of the matrix $\varepsilon(A)^{t}=\left[\varepsilon\left(p_{i j}(t)\right)\right]$ are relatively prime.

ExAmple 4.5. Consider $K^{2}=T \vee \bigvee_{i=1}^{n} S^{2}$, where $T$ is the 2-torus. Its universal cover $\widetilde{K}^{2}$ is $\mathbb{R}^{2}$ together with a copy of $\bigvee_{i=1}^{n} S^{2}$ attached to each point $(x, y)$ with integral coordinates (see Figure 1 ). Contracting $\mathbb{R}^{2}$ to a point, we can see that $\pi_{2}\left(K^{2}\right)=H_{2}\left(\widetilde{K}^{2}\right)=\bigoplus_{i=1}^{n} \mathbb{Z}\left[x, y, x^{-1}, y^{-1}\right]$. Therefore each element of $\pi_{2}\left(K^{2}\right)$ is represented by an $n$-tuple $\left(p_{1}(x, y), \ldots, p_{n}(x, y)\right)$ of Laurent polynomials in two variables. As above, to construct from $K^{2}$ complexes $K$ with $H^{3}(K ; \mathbb{Z})=0$, it suffices to attach $m$ 3-cells with $m \leq n$ for polynomials such that the matrix $\varepsilon(A)^{t}=\left[\varepsilon\left(p_{i j}(x, y)\right)\right]$ has its $m \times m$ minors relatively prime.

REMARK 4.6. Observe that $T$ can be substituted by any compact orientable surface, $S_{g}$, of genus $g \geq 1$.

To construct a complex of dimension $r$, we have to attach cells via maps $\varphi: S^{r-1} \rightarrow K^{r-1}$ to a complex of dimension $r-1$. But these maps represent elements of $\pi_{r-1}\left(K^{r-1}\right)$. In the case $r=3$, we have a tool to calculate $\pi_{2}\left(K^{2}\right)$ for any connected 2-complex. This technique is presented in detail in [HMS, Chapter 2]. We will describe it as follows. 


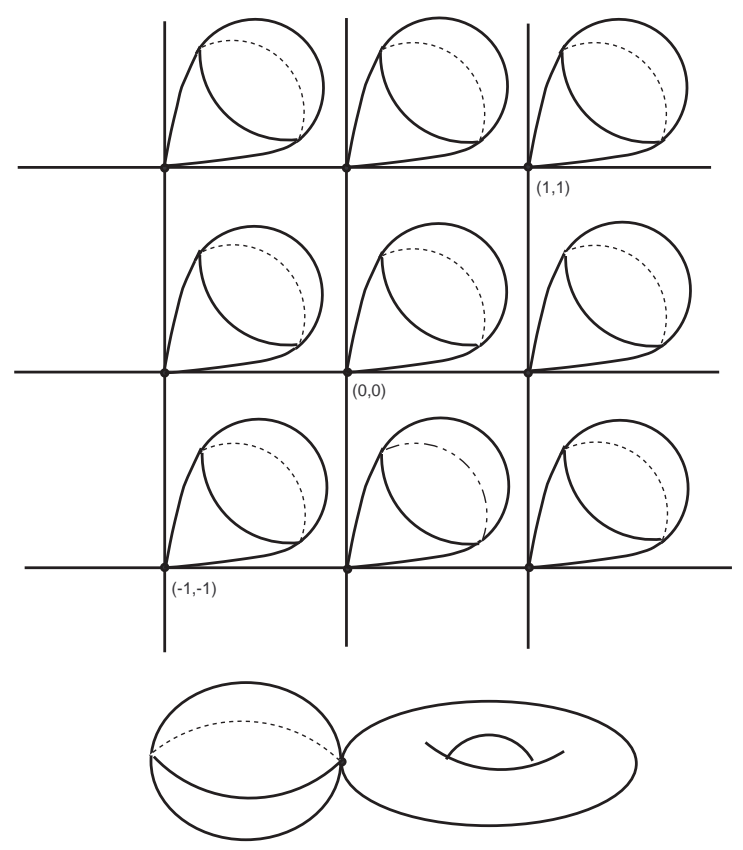

Fig. 1

All 1-dimensional connected $C W$-complexes $K^{1}$ have the homotopy type of a bouquet $\bigvee_{i=1}^{n} S^{1}$. Its fundamental group is

$$
\pi_{1}\left(\bigvee_{i=1}^{n} S^{1}\right)=F\left(x_{1}, \ldots, x_{n}\right)=\text { free group on } n \text { generators. }
$$

The 2-complexes $K^{2}$ are obtained from $K^{1}$ by attaching $p$ cells of dimension 2, via maps $S^{1} \rightarrow \bigvee_{i=1}^{n} S^{1}$. But these maps represent elements of $\pi_{1}\left(K^{1}\right)$. So, the fundamental group of any connected 2-complex $K^{2}$ can be given a presentation $\left\langle x_{1}, \ldots, x_{n} \mid r_{1}, \ldots, r_{p}\right\rangle$, where $r_{i}$ are the relations defined by the homotopy classes of the attaching maps. Therefore

$$
\pi_{1}\left(K^{2}\right)=F\left(x_{1}, \ldots, x_{n}\right) / N\left(r_{1}, \ldots, r_{p}\right),
$$

where $N\left(r_{1}, \ldots, r_{p}\right)$ is the smallest normal subgroup generated by $r_{1}, \ldots, r_{p}$. Denote by

$$
|-|: F(x)=F\left(x_{1}, \ldots, x_{n}\right) \rightarrow F\left(x_{1}, \ldots, x_{n}\right) / N\left(r_{1}, \ldots, r_{p}\right)
$$

the projection. Let $\widetilde{K}^{2}$ be the universal covering of $K^{2}$. The boundary operator

$$
\widetilde{\partial}_{2}: H_{2}\left(\widetilde{K}^{2}, \widetilde{K}^{1}\right) \rightarrow H_{1}\left(\widetilde{K}^{1}, \widetilde{K}^{0}\right)
$$


is obtained in the following way: for each $x_{i}$ consider Fox's derivative

$$
\frac{\partial}{\partial x_{i}}: F(x) \rightarrow \mathbb{Z} F(x)
$$

which is the only map satisfying

$$
\frac{\partial w_{1} w_{2}}{\partial x_{i}}=\frac{\partial w_{1}}{\partial x_{i}}+w_{1} \frac{\partial w_{2}}{\partial x_{i}} \quad \text { for all } w_{1}, w_{2} \in F(x),
$$

and whose value at $x_{j}$ is $\delta_{i, j}$ (Kronecker delta). Then we have

$$
\widetilde{\partial}_{2}\left(\widetilde{e}_{r_{j}}^{2}\right)=\sum_{i=1}^{n}\left\|\frac{\partial r_{j}}{\partial x_{i}}\right\| \widetilde{e}_{x_{i}}^{1} \quad \text { for } j=1, \ldots, p .
$$

Here $\|-\|: \mathbb{Z} F(x) \rightarrow \mathbb{Z} \pi_{1}$ is the natural extension of the projection $|-|$.

From the exact sequence

$$
0 \rightarrow H_{2}\left(\widetilde{K}^{2}\right) \stackrel{j_{*}}{\longrightarrow} H_{2}\left(\widetilde{K}^{2}, \widetilde{K}^{1}\right) \stackrel{\widetilde{\partial}_{2}}{\longrightarrow} H_{1}\left(\widetilde{K}^{1}, \widetilde{K}^{0}\right)
$$

we obtain $\pi_{2}\left(K^{2}\right)=H_{2}\left(\widetilde{K}^{2}\right)=\operatorname{Ker} \widetilde{\partial}_{2}$.

EXAmPle 4.7. Let $K^{2}$ be a 2-complex with $\pi_{1}\left(K^{2}\right)=\left\langle x, y \mid x^{3}, y^{2},(x y)^{2}\right\rangle$ (this is a presentation of the dihedral group $D_{3}$ ). The homomorphism $\widetilde{\partial}_{2}$ : $H_{2}\left(\widetilde{K}^{2}, \widetilde{K}_{1}\right) \rightarrow H_{1}\left(\widetilde{K}_{1}, \widetilde{K}_{0}\right)$ is given by the matrix

$$
\left[\begin{array}{ccc}
1+x+x^{2} & 0 & 1+x y \\
0 & 1+y & x+y
\end{array}\right]
$$

and the vectors

$$
\left[-1-y,-1-x-x^{2}, y+x y+x^{2} y\right]
$$

and

$$
\left[-1-y,-1-x-x^{2}, 1+x+x^{2}\right]
$$

belong to the kernel of $\widetilde{\partial}_{2}$, and hence represent elements of $\pi_{2}\left(K^{2}\right)$. If $K$ is obtained from $K^{2}$ by gluing one 3 -cell through one of those two maps, then $H^{3}(K, \mathbb{Z})=0$.

Observe that, if $K^{2}$ is a 2-complex having $n$ cells of dimension 2 and $m$ cells of dimension 3 are attached via elements $\alpha_{i}=\left[\widetilde{a}_{1 i}, \ldots, \widetilde{a}_{n i}\right] \in \operatorname{Ker} \widetilde{\partial}_{2}$ for $i=1, \ldots, m$, then the boundary operator $\widetilde{\partial}_{3}: H_{3}\left(\widetilde{K}^{3}, \widetilde{K}^{2}\right) \rightarrow H_{2}\left(\widetilde{K}^{2}, \widetilde{K}^{1}\right)$ is given by

$$
\widetilde{\partial}_{3}\left(\widetilde{e}_{i}^{3}\right)=\widetilde{a}_{1 i} \widetilde{e}_{1}^{2}+\cdots+\widetilde{a}_{n i} \widetilde{e}_{n}^{2},
$$

where $\widetilde{e}_{i}^{3}$ is the cell that corresponds to the attaching map $\alpha_{i}$. For a left 
$\mathbb{Z}[\Pi]$-module $A$, we have a commutative diagram

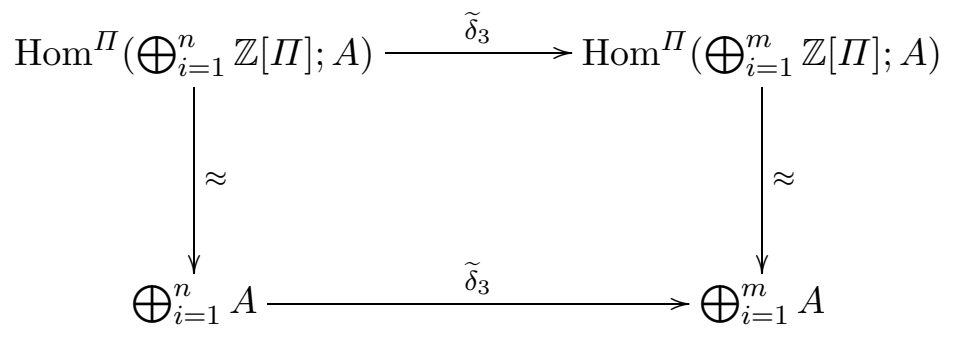

where for $b=\left[b_{1}, \ldots, b_{n}\right] \in \bigoplus_{i=1}^{n} A$, the vector $\widetilde{\delta}_{3}(b) \in \bigoplus_{i=1}^{m} A$ is given by the product

$$
\left[\begin{array}{ccc}
\widetilde{a}_{11} & \ldots & \widetilde{a}_{n 1} \\
\vdots & \vdots & \vdots \\
\widetilde{a}_{1 m} & \ldots & \widetilde{a}_{n m}
\end{array}\right]\left[\begin{array}{c}
b_{1} \\
\vdots \\
b_{n}
\end{array}\right] .
$$

5. Manifolds with abelian fundamental group. Assume $M$ is a 3manifold with a $C W$-decomposition with only one cell, $e_{M}$, of dimension 3 . In order to decide if $\operatorname{MR}[f, a]$ vanishes, from 3.8 we may assume that $f$ : $K \rightarrow M$ is cellular and we must consider $\omega^{3}(f) \in H^{3}\left(K ; f^{*} \pi_{3}\left(M, M^{2}\right)\right)$.

Let $q: \widetilde{M} \rightarrow M$ be the universal covering of $M$ and $m_{0}$ a base point. Consider the following local coefficient system on $M$. For each $\alpha \in \pi_{1}(M)$ let $h_{\alpha}: \widetilde{M} \rightarrow \widetilde{M}$ be the homeomorphism satisfying $q \circ h_{\alpha}=q$ and $h_{\alpha}\left(m_{0}\right)=$ $\widetilde{\alpha}(1)$, where $\widetilde{\alpha}$ is the lift of $\alpha$ beginning at $m_{0}$. Define $\alpha x=\left(h_{\alpha}\right)_{*}(x)$ for all $x \in H_{3}\left(\widetilde{M}, \widetilde{M}^{2}\right)$, where $\left(h_{\alpha}\right)_{*}: H_{3}\left(\widetilde{M}, \widetilde{M}^{2}\right) \rightarrow H_{3}\left(\widetilde{M}, \widetilde{M}^{2}\right)$ is the induced isomorphism. Call this system $\mathcal{R}$. Notice that $H_{3}\left(\widetilde{M}, \widetilde{M}^{2}\right)=\mathbb{Z}[\pi]$, where $\pi=$ $\pi_{1}(M)$, and the action is given by the homomorphism $\Omega: \pi \rightarrow \operatorname{Aut}(\mathbb{Z}[\pi])$ where $\Omega(\theta)(\alpha)=\theta \alpha$. Now consider $f^{*} \mathcal{R}$, the local coefficient system over $K$ induced by $f_{\#}: \pi_{1}(K) \rightarrow \pi_{1}(M)$.

Lemma 5.1. The local systems $\mathcal{R}$ and $\pi_{3}\left(M, M^{2}\right)$ are isomorphic.

Proof. Consider the commutative diagram

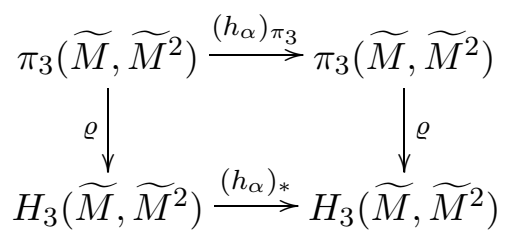

The Hurewicz homomorphism is a homomorphism between the systems $\mathcal{R}$ and $\pi_{3}\left(M, M^{2}\right)$. Since $H_{p}\left(\widetilde{M}, \widetilde{M}^{2}\right)=0$ for $p<3$ and $\pi_{1}\left(\widetilde{M}^{2}\right)=0$, the Hurewicz homomorphism is an isomorphism.

It follows that $\omega^{3}(f)=0$ if, and only if, $\varrho_{*}\left(\omega^{3}(f)\right) \in H^{3}\left(K ; f^{*} \mathcal{R}\right)$ is zero. 
REMARK 5.2. To simplify the notation, we will write $H^{3}(K ; \mathbb{Z}[\pi])$ for the group $H^{3}\left(K ; f^{*} \pi_{3}\left(M, M^{2}\right)\right)$. Also we will use the same symbol $f_{\#}$ for its extension $\left(f_{\#}\right)_{\mathbb{Z}}: \mathbb{Z}[\Pi] \rightarrow \mathbb{Z}[\pi]$.

Theorem 5.3. Assume that $\widetilde{\partial}_{3}: H_{3}\left(\widetilde{K}^{3}, \widetilde{K}^{2}\right) \rightarrow H_{2}\left(\widetilde{K}^{2}, \widetilde{K}^{1}\right)$ is of the form

$$
\widetilde{\partial}_{3}\left(\widetilde{e}_{i}^{3}\right)=\widetilde{a}_{1 i} \widetilde{e}_{1}^{2}+\cdots+\widetilde{a}_{n i} \widetilde{e}_{n}^{2}
$$

for $i=1, \ldots, m$. The homomorphism $\psi \in \operatorname{Hom}^{\Pi}\left(\bigoplus_{i=1}^{m} \mathbb{Z}[\Pi] ; \mathbb{Z}[\pi]\right)$, given by $\psi\left(\widetilde{e}_{i}^{3}\right)=k_{i}$ for $i=1, \ldots, m$ belongs to the image of

$$
\widetilde{\delta}_{3}: \operatorname{Hom}^{\Pi}\left(C_{2}(\widetilde{K}) ; \mathbb{Z}[\pi]\right) \rightarrow \operatorname{Hom}^{\Pi}\left(C_{3}(\widetilde{K}) ; \mathbb{Z}[\pi]\right)
$$

if, and only if, the system $P x=k$ has a solution in $\mathbb{Z}[\pi]$, where

$$
P=\left[\begin{array}{ccc}
f_{\#}\left(\widetilde{a}_{11}\right) & \ldots & f_{\#}\left(\widetilde{a}_{n 1}\right) \\
\vdots & \vdots & \vdots \\
f_{\#}\left(\widetilde{a}_{1 m}\right) & \ldots & f_{\#}\left(\widetilde{a}_{n m}\right)
\end{array}\right], \quad k=\left[k_{1}, \ldots, k_{m}\right]^{t} .
$$

Proof. $\psi \in \operatorname{Im} \widetilde{\delta}_{3}$ if, and only if, there exists $\phi \in \operatorname{Hom}^{\Pi}\left(\bigoplus_{i=1}^{n} \mathbb{Z}[\Pi] ; \mathbb{Z}[\pi]\right)$ such that $\widetilde{\delta}_{3}(\phi)=\phi \circ \widetilde{\partial}_{3}=\psi$. Notice that $\phi\left(\widetilde{e}_{j}^{2}\right)=x_{j}$ for $j=1, \ldots, n$. So $\phi\left(\widetilde{a}_{1 i} \widetilde{e}_{1}^{2}+\cdots+\widetilde{a}_{n i} \widetilde{e}_{n}^{2}\right)=\widetilde{a}_{1 i} \phi\left(\widetilde{e}_{1}^{2}\right)+\cdots+\widetilde{a}_{n i} \phi\left(\widetilde{e}_{n}^{2}\right)$ is also equal to $f_{\#}\left(\widetilde{a}_{1 i}\right) x_{1}+\cdots+f_{\#}\left(\widetilde{a}_{n i}\right) x_{n}$ for $i=1, \ldots, m$ with respect to the action above. Equivalently,

$$
\left[\begin{array}{ccc}
f_{\#}\left(\widetilde{a}_{11}\right) & \ldots & f_{\#}\left(\widetilde{a}_{n 1}\right) \\
\vdots & \vdots & \vdots \\
f_{\#}\left(\widetilde{a}_{1 m}\right) & \ldots & f_{\#}\left(\widetilde{a}_{n m}\right)
\end{array}\right]\left[\begin{array}{c}
x_{1} \\
\vdots \\
x_{n}
\end{array}\right]=\left[\begin{array}{c}
k_{1} \\
\vdots \\
k_{m}
\end{array}\right] .
$$

LEMmA 5.4. The obstruction class $\varrho_{*}\left(\omega^{3}(f)\right)$ is represented by the cochain

$$
\widetilde{f}_{*}: H_{3}\left(\widetilde{K}^{3}, \widetilde{K}^{2}\right) \rightarrow H_{3}\left(\widetilde{M}, \widetilde{M}^{2}\right) .
$$

Proof. Recall that $\omega^{3}(f)$ is represented by the cochain $c^{3}(f)$ defined by

$$
c^{3}(f)=\widetilde{f}_{\pi_{3}} \circ \varrho^{-1}: H_{3}\left(\widetilde{K}^{3}, \widetilde{K}^{2}\right) \rightarrow \pi_{3}\left(\widetilde{K}^{3}, \widetilde{K}^{2}\right) \rightarrow \pi_{3}\left(\widetilde{M}, \widetilde{M}^{2}\right) .
$$

From the commutative diagram

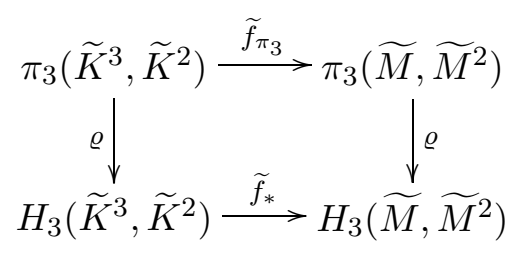

the cochain representing the class $\varrho_{*}\left(\omega^{3}(f)\right)$ is $\varrho_{\#}\left(c^{3}(f)\right)=c^{3}(f) \circ \varrho=\widetilde{f}_{*}$. 
COROLlary 5.5. The obstruction class $\varrho_{*}\left(\omega^{3}(f)\right)$ is zero if, and only if, the system $P x=k$ has a solution in $\mathbb{Z}[\pi]$, where $k=\left[\widetilde{f}_{*}\left(\widetilde{e}_{1}^{3}\right), \ldots, \widetilde{f}_{*}\left(\widetilde{e}_{m}^{3}\right)\right]^{t}$, and $P$ is the matrix given in Theorem 5.3 .

Proof. The vanishing of $\varrho_{*}\left(\omega^{3}(f)\right)$ means that the cochain representing this cohomology class is in the image of $\widetilde{\delta}_{3}$. Now the result follows from Lemma 5.4 and Theorem 5.3.

THEOREM 5.6. The cochain obstruction $\widetilde{f}_{*}: H_{3}\left(\widetilde{K}^{3}, \widetilde{K}^{2}\right) \rightarrow H_{3}\left(\widetilde{M}, \widetilde{M}^{2}\right)$ has the property that the system $P x=\widetilde{f}_{*}\left(\widetilde{e}_{i}^{3}\right) \widetilde{\partial}_{M}\left(\widetilde{e}_{M}\right)$ for $i=1, \ldots, m$ has a solution in the $\mathbb{Z}[\pi]$-module $H_{2}\left(\widetilde{M}^{2}, \widetilde{M}^{1}\right)$. Here

$$
\widetilde{\partial}_{M}: H_{3}\left(\widetilde{M}, \widetilde{M}^{2}\right) \rightarrow H_{2}\left(\widetilde{M}^{2}, \widetilde{M}^{1}\right)
$$

is the boundary homomorphism.

Proof. Since $f: K \rightarrow M$ is a cellular map, the diagram

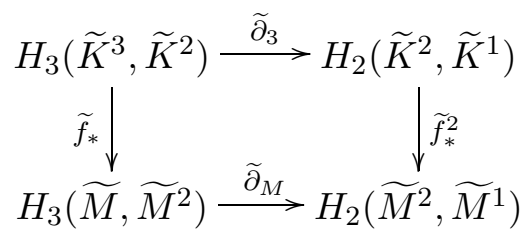

is commutative. Hence

$$
\begin{aligned}
\widetilde{f}_{*}\left(\widetilde{e}_{i}^{3}\right) \widetilde{\partial}_{M}\left(\widetilde{e}_{M}\right) & =\widetilde{\partial}_{M}\left(f_{*}\left(\widetilde{e}_{i}^{3}\right) \widetilde{e}_{M}\right)=\left(\widetilde{\partial}_{M} \circ \widetilde{f}_{*}\right)\left(\widetilde{e}_{i}^{3}\right) \\
& =\left(\widetilde{f}_{*}^{2} \circ \widetilde{\partial}_{3}\right)\left(\widetilde{e}_{i}^{3}\right)=\widetilde{f}_{*}^{2}\left(\widetilde{a}_{1 i} \widetilde{e}_{1}^{2}+\cdots+\widetilde{a}_{n i} \widetilde{e}_{n}^{2}\right) \\
& =\widetilde{f}_{*}^{2}\left(\widetilde{a}_{1 i} \widetilde{e}_{1}^{2}\right)+\cdots+\widetilde{f}_{*}^{2}\left(\widetilde{a}_{n i} \widetilde{e}_{n}^{2}\right) \\
& =f_{\#}\left(\widetilde{a}_{1 i}\right) \widetilde{f}_{*}^{2}\left(\widetilde{e}_{1}^{2}\right)+\cdots+f_{\#}\left(\widetilde{a}_{n i}\right) \widetilde{f}_{*}^{2}\left(\widetilde{e}_{n}^{2}\right),
\end{aligned}
$$

that is,

$$
\left[\begin{array}{ccc}
f_{\#}\left(\widetilde{a}_{11}\right) & \ldots & f_{\#}\left(\widetilde{a}_{n 1}\right) \\
\vdots & \vdots & \vdots \\
f_{\#}\left(\widetilde{a}_{1 m}\right) & \ldots & f_{\#}\left(\widetilde{a}_{n m}\right)
\end{array}\right]\left[\begin{array}{c}
\widetilde{f}_{*}^{2}\left(\widetilde{e}_{1}^{2}\right) \\
\vdots \\
\widetilde{f}_{*}^{2}\left(\widetilde{e}_{n}^{2}\right)
\end{array}\right]=\left[\begin{array}{c}
\widetilde{f}_{*}\left(\widetilde{e}_{1}^{3}\right) \widetilde{\partial}_{M}\left(\widetilde{e}_{M}\right) \\
\vdots \\
\widetilde{f}_{*}\left(\widetilde{e}_{m}^{3}\right) \widetilde{\partial}_{M}\left(\widetilde{e}_{M}\right)
\end{array}\right] \cdot \cdot \cdot
$$

THEOREM 5.7. Let $K$ be a finite 3-complex. Then there is no map $f$ : $K \rightarrow S^{1} \times S^{2}$ which is strongly surjective if one of the following conditions holds:

(i) $H^{3}(K ; \mathbb{Z})=0$,

(ii) $H^{3}(K ; \mathbb{Z})$ is finite and $H^{3}\left(K ; \mathbb{Z}\left[t, t^{-1}\right]\right)$ torsion free,

(iii) $K$ is square and $H^{3}(K ; \mathbb{Z})$ finite.

Proof. Consider $S^{1} \times S^{2}$ as a $C W$-complex with 1-skeleton being $S^{1}$, 2-skeleton being $M^{2}=S^{1} \vee S^{2}$, and $S^{1} \times S^{2}$ is obtained from $M^{2}$ by attaching 


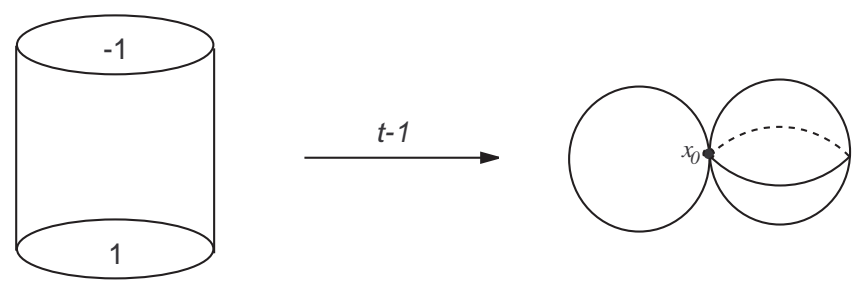

Fig. 2

a 3-cell via the map $t-1 \in \pi_{2}\left(M^{2}\right)=\mathbb{Z}\left[t, t^{-1}\right]$. Geometrically the map $t-1$ can be regarded as a map from the cylinder which is the boundary of $D^{2} \times I$ (see Fig. 2) into $S^{1} \vee S^{2}$. This map restricted to the top disc $D^{2} \times\{1\}$ sends the boundary of the disc to the point $x_{0}$ and has degree one. On $\partial D^{2} \times I$ the map is the composite of the projection $\partial D^{2} \times I \rightarrow I$ followed by the maps $I \rightarrow S^{1} \rightarrow S^{1} \vee S^{2}$ where the first map sends $\{0,1\} \rightarrow x_{0}$ and it is of degree one. On the bottom disc $D^{2} \times\{0\}$, the map sends the boundary of the disc to the point $x_{0}$ and has degree -1 . The boundary operator

$$
\widetilde{\partial}_{S^{1} \times S^{2}}: \mathbb{Z}\left[t, t^{-1}\right] \rightarrow \mathbb{Z}\left[t, t^{-1}\right]
$$

is given by $\widetilde{\partial}_{S^{1} \times S^{2}}(b)=(t-1) b$.

(i) We have to show that the system $P x=k$ has a solution in $\mathbb{Z}\left[t, t^{-1}\right]$, where $k_{i}=\widetilde{f}_{*}\left(\widetilde{e}_{i}^{3}\right)$ for $i=1, \ldots, m$. From Theorem 5.6, we know that $P x=$ $(t-1) k$ has the vector $e=\left[\widetilde{f}_{*}^{2}\left(\widetilde{e}_{1}^{2}\right), \ldots, \widetilde{f}_{*}^{2}\left(\widetilde{e}_{n}^{2}\right)\right]^{t}$ as a solution. Let $d_{1}, \ldots, d_{r}$ be all $m \times m$ minors of $P$. The fact that $H^{3}(K ; \mathbb{Z})=0$ means that the integers $\varepsilon\left(d_{1}\right), \ldots, \varepsilon\left(d_{r}\right)$ are relatively prime. From Lemma 2.3 it follows that $P x=k$ has a solution.

(ii) The fact that $H^{3}(K ; \mathbb{Z})$ is finite implies that $\varepsilon\left(d_{i}\right) \neq 0$ (see [BR2, Chapter 3, Proposition 15]) for some $i=1, \ldots, r$. The system $P x=d_{i} k$ has a vector $y$ as a solution. Let $q \in \mathbb{Z}\left[t, t^{-1}\right]$ be such that $d_{i}-q(t-1)=\varepsilon\left(d_{i}\right)$. Hence $P(y-q e)=P y-P q e=d_{i} k-q k(t-1)=\left(d_{i}-q(t-1)\right) k=\varepsilon\left(d_{i}\right) k$. This means that the obstruction class $\varrho_{*}\left(\omega^{3}(f)\right)$ is a torsion element of $H^{3}\left(K ; \mathbb{Z}\left[t, t^{-1}\right]\right)$. Since this group is torsion free, the obstruction class is zero.

(iii) If $K$ is square, then $P$ is a square matrix. If $H^{3}(K ; \mathbb{Z})$ is finite, then $\varepsilon(\operatorname{det}(P)) \neq 0$, and this forces the vector solution $e$ of the system $P x=k(t-1)$ to have the property that $\varepsilon\left(\widetilde{f}_{*}^{2}\left(\widetilde{e}_{i}^{2}\right)\right)=0$ for all $i=1, \ldots, n$. But this means that $e=(t-1) a$, where $a=\left[a_{1}, \ldots, a_{n}\right]^{t}$ is a vector with coordinates in $\mathbb{Z}\left[t, t^{-1}\right]$. Hence $P a=k$.

Consider the manifold

$$
M_{A}=S^{2} \times[0,1] /(x, 0) \sim(-x, 1) .
$$


To get a $C W$-decomposition of $M_{A}$, it is enough to attach a 3 -cell to $S^{1} \vee S^{2}$ via the map $t+1$. The boundary operator

$$
\widetilde{\partial}_{M_{A}}: \mathbb{Z}\left[t, t^{-1}\right] \rightarrow \mathbb{Z}\left[t, t^{-1}\right]
$$

is given by $\widetilde{\partial}_{M_{A}}(b)=(t+1) b$. The fact that $H^{3}(K ; \mathbb{Z})=0$ is not enough to guarantee that $\operatorname{MR}[f, a]=0$ for all $f: K \rightarrow M_{A}$.

TheOREM 5.8. There exist a 3 -complex $K$ with $H^{3}(K ; \mathbb{Z})=0$ and a map $f: K \rightarrow M_{A}$ which is strongly surjective.

Proof. Let $K^{2}=S^{1} \vee \bigvee_{i=1}^{2} S^{2}$, and $K$ the complex obtained from $K^{2}$ by attaching a 3 -cell via the map $\left(2 t^{2}+2 t, 2 t^{2}+1\right)$. From Example 4.4 , we have $H^{3}(K ; \mathbb{Z})=0$.

Define $f: S^{1} \vee \bigvee_{i=1}^{2} S^{2} \rightarrow S^{1} \vee S^{2}$ on the 2-skeleton of $K$ to the 2-skeleton of $M_{A}$, as:

- $f \mid S^{1}$ is the identity map on $S^{1}$.

- $f\left(e_{1}^{2}\right)=3 t+2$ and $f\left(e_{2}^{2}\right)=t+1$.

Since $\left(2 t^{2}+2 t\right)(3 t+2)+\left(2 t^{2}+1\right)(t+1)=k \cdot(t+1), f$ extends to $K$. Note that $\widetilde{f}_{*}\left(\widetilde{e}^{3}\right)=k(t)=8 t^{2}+4 t+1$, so $k(-1)=5$. This shows that $k$ is not of the form $\left(2 t^{2}+2 t\right) a+\left(2 t^{2}+1\right) b$. Therefore all elements of this form, when $t=-1$, are integers divisible by 3 .

Let $f_{\#}: \pi_{1}(K) \rightarrow \pi_{1}\left(M_{A}\right)=\mathbb{Z}$ and $\widetilde{\mathbb{Z}}$ be the local coefficient system over $K$, given by the action of $\pi_{1}(K)$ on $\mathbb{Z}$ : for $\alpha \in \pi_{1}(K)$,

$$
\alpha 1= \begin{cases}1 & \text { if } f_{\#}(\alpha)=t^{k} \text { with } k \text { even }, \\ -1 & \text { if } f_{\#}(\alpha)=t^{k} \text { with } k \text { odd } .\end{cases}
$$

Theorem 5.9. Let $K$ be a finite 3-complex. There is no map $f: K \rightarrow$ $M_{A}$ which is strongly surjective if one of the following conditions holds:

(i) $H^{3}(K ; \widetilde{\mathbb{Z}})=0$,

(ii) $H^{3}(K ; \widetilde{\mathbb{Z}})$ is finite and $H^{3}\left(K ; \mathbb{Z}\left[t, t^{-1}\right]\right)$ torsion free,

(iii) $K$ is square and $H^{3}(K ; \widetilde{\mathbb{Z}})$ finite.

Proof. (i) As before we have to show that the system $P x=k$ has a solution, where $k=\left[\widetilde{f}_{*}\left(\widetilde{e}_{1}^{3}\right), \ldots, \widetilde{f}_{*}\left(\widetilde{e}_{m}^{3}\right)\right]^{t}$. From Theorem 5.6, we know that the system $P x=k(t+1)$ has the vector $e=\left[\widetilde{f}_{*}^{2}\left(\widetilde{e}_{1}^{2}\right), \ldots, \widetilde{f}_{*}^{2}\left(\widetilde{e}_{n}^{2}\right)\right]^{t}$ as a solution. From the proof of Lemma 3.3, the fact that $H^{3}(K ; \widetilde{\mathbb{Z}})=0$ implies that the $m \times m$ minors of $P$ have the property that $\bar{\varepsilon}\left(d_{1}\right), \ldots, \bar{\varepsilon}\left(d_{r}\right)$ are relatively prime. From Lemma 2.2 , there exist integers $p_{1}, \ldots, p_{r}$ and $q \in$ $\mathbb{Z}\left[t, t^{-1}\right]$ such that $p_{1} d_{1}+\cdots+p_{r} d_{r}-q(t+1)=1$. Since the system $P x=d_{i} k$ has a solution for $i=1, \ldots, r$, and no maximal ideal $\mathfrak{m}$ can contain the set $\left\{d_{1}, \ldots, d_{r}, t+1\right\}$, by [HG, Proposition 1] the system $P x=k$ has a solution. 
(ii) When $H^{3}(K ; \widetilde{\mathbb{Z}})$ is finite, it follows that $\bar{\varepsilon}\left(d_{i}\right) \neq 0$ for some $i=$ $1, \ldots, r$. Let $q \in \mathbb{Z}\left[t, t^{-1}\right]$ be such that $d_{i}-q(t+1)=\bar{\varepsilon}\left(d_{i}\right)$, and $y$ be the vector solution of the equation $P x=d_{i} k$. Then $P(y-q e)=P y-P q e=$ $d_{i} k-q(t+1) k=\left(d_{i}-q(t+1)\right) k=\bar{\varepsilon}\left(d_{i}\right) k$, which means that the obstruction class $\varrho_{*}\left(\omega^{3}(f)\right)$ is a torsion element. Since $H^{3}\left(K ; \mathbb{Z}\left[t, t^{-1}\right]\right)$ is torsion free, $\varrho_{*}\left(\omega^{3}(f)\right)=0$.

(iii) When $P$ is a square matrix and $H^{3}(K ; \widetilde{\mathbb{Z}})$ is finite, we have $\bar{\varepsilon}(\operatorname{det}(P))$ $\neq 0$, which forces $\bar{\varepsilon}\left(\widetilde{f}_{*}^{2}\left(\widetilde{e}_{1}^{2}\right)\right)=0, \ldots, \bar{\varepsilon}\left(\widetilde{f}_{*}^{2}\left(\widetilde{e}_{n}^{2}\right)\right)=0$, or equivalently, $e=$ $a(t+1)$ and $P a=k$.

REMARK 5.10. As a consequence of Lemma 3.3, the assumption that $H^{3}(K ; \widetilde{\mathbb{Z}})$ is finite in (ii) and (iii) may be replaced by $H^{3}(K ; \mathbb{Z})$ being finite of odd cardinality.

Let $n$ and $p$ be relatively prime positive integers. The symbol $L(n, p)$ denotes the lens space. In [M, Chapter 4], it is proved that $M=L(n, p)$ has a cellular decomposition with a cell in each dimension $0,1,2,3$, and its 2-skeleton is $M^{2}$. Since the universal cover of $L(n, p)$ is $S^{3}$, the sequence

$$
0 \rightarrow H_{3}\left(\widetilde{M}{ }^{3}, \widetilde{M}^{2}\right) \stackrel{\widetilde{\partial}_{3}}{\longrightarrow} H_{2}\left(\widetilde{M}^{2}, \widetilde{M}^{1}\right) \stackrel{\widetilde{\partial}_{2}}{\longrightarrow} H_{1}\left(\widetilde{M}^{1}, \widetilde{M}^{0}\right) \stackrel{\widetilde{\partial}_{1}}{\longrightarrow} H_{0}\left(\widetilde{M}^{0}\right) \rightarrow 0
$$

gives the homology of the sphere, and therefore $\operatorname{Im} \widetilde{\partial}_{3}=\operatorname{Ker} \widetilde{\partial}_{2}$. But the operator $\widetilde{\partial}_{2}: \mathbb{Z}\left[\mathbb{Z}_{n}\right] \rightarrow \mathbb{Z}\left[\mathbb{Z}_{n}\right]$ is given by the Fox derivative, that is, $\widetilde{\partial}_{2}\left(\widetilde{e}^{2}\right)=$ $1+x+\cdots+x^{n-1}$, and its kernel is the ideal generated by $x-1$. Therefore $\widetilde{\partial}_{3}\left(\widetilde{e}^{3}\right)=x-1$.

TheOREM 5.11. Let $K$ be a finite 3-complex. There is no map $f: K \rightarrow$ $M=L(n, p)$ which is strongly surjective if one of the following conditions holds:

(i) $H^{3}(K ; \mathbb{Z})=0$,

(ii) $H^{3}(K ; \mathbb{Z})$ is finite and $H^{3}\left(K ; \mathbb{Z}\left[\mathbb{Z}_{n}\right]\right)$ torsion free.

Proof. (i) The proof is similar to the proof of Theorem 5.9(i). The system $P x=k(x-1)$ has a solution and we want to know if $P x=k$ has a solution. However $H^{3}(K ; \mathbb{Z})=0$ implies that the integers $\varepsilon\left(d_{1}\right), \ldots, \varepsilon\left(d_{r}\right)$ are relatively prime and so the set $\left\{d_{1}, \ldots, d_{r}, x-1\right\}$ cannot belong to a maximal ideal $\mathfrak{m}$. From $[\mathrm{HG}$, Proposition 1] the system $P x=k$ has a solution.

(ii) The obstruction class is a torsion element of $H^{3}\left(K ; \mathbb{Z}\left[\mathbb{Z}_{n}\right]\right)$. Therefore it must be zero.

REMARK 5.12. In case $H^{3}(K ; \mathbb{Z})$ is finite and $K$ is square, it is not possible to guarantee that the system has a solution. As before we will have $e=a(x-1)$. However, this implies that the system

$$
P x=k+\left[p_{1}, \ldots, p_{n}\right]^{t}\left(1+x+\cdots+x^{n-1}\right)
$$

has a solution for some vector $\left[p_{1}, \ldots, p_{n}\right]^{t}$ with integer coordinates. 
Corollary 5.13. If $f: M_{A} \rightarrow \mathbb{P}^{3}$ has $f_{\#}: \pi_{1}\left(M_{A}\right) \rightarrow \pi_{1}\left(\mathbb{P}^{3}\right)$ surjective, then $f$ is strongly surjective.

Proof. We know that $M_{A}$ is obtained by attaching a 3-cell via the map $t+1$ to the bouquet $S^{1} \vee S^{2}$, and that $H^{3}\left(M_{A} ; \mathbb{Z}\right)=\mathbb{Z}_{2}$. Since $f_{\#}$ is surjective, $f_{\#}(t)=x$ and we have

$$
H^{3}\left(M_{A} ; \mathbb{Z}\left[\mathbb{Z}_{2}\right]\right)=\mathbb{Z}\left[\mathbb{Z}_{2}\right] /(x+1)=\mathbb{Z} .
$$

From Theorem 5.11 it follows that $\operatorname{MR}[f, a]=0$.

Consider the manifold $S^{1} \times \mathbb{P}^{2}$. Its $C W$-decomposition is obtained in the following way: Its 1-skeleton is $K^{1}=\bigvee_{i=1}^{2} S_{i}^{1}$, a bouquet of two circles, the 2-skeleton $K^{2}$ is the union of a torus $T$ with a projective space $\mathbb{P}^{2}$, and its universal cover $\widetilde{K}^{2}$ is the infinite cylinder $\mathbb{R} \times S^{1}$, where a sphere $S^{2}$ is attached to each $\{n\} \times S^{1}$ for any integer $n$. The covering map $p: \widetilde{K}^{2} \rightarrow K^{2}$ is the projection map $S^{2} \rightarrow \mathbb{P}^{2}$ on each $S^{2}$, and the map $g(z, t)=\left(z^{2}, t\right)$ on $\mathbb{R} \times S^{1}$ (see Figure 3). Finally, $S^{1} \times \mathbb{P}^{2}$ is obtained from $K^{2}$ by attaching a 3-cell via the map $p \mid D^{2} \times[0,1]: D^{2} \times[0,1] \rightarrow K_{2}$. The boundary operator $\widetilde{\partial}_{S^{1} \times \mathbb{P}^{2}}:$ $\mathbb{Z}\left[\mathbb{Z} \times \mathbb{Z}_{2}\right] \rightarrow \mathbb{Z}\left[\mathbb{Z} \times \mathbb{Z}_{2}\right] \oplus \mathbb{Z}\left[\mathbb{Z} \times \mathbb{Z}_{2}\right]$ is given by $\widetilde{\partial}_{S^{1} \times \mathbb{P}^{2}}(b)=b(x+1, y-1)$.

For a map $f: K \rightarrow S^{1} \times \mathbb{P}^{2}$, consider the system $\widetilde{\mathbb{Z}}$ given by the action of $\pi_{1}(K)$ on $\mathbb{Z}$ defined by

$$
\alpha 1= \begin{cases}-1 & \text { if } f_{\#}(\alpha)=y^{k} x \\ 1 & \text { if } f_{\#}(\alpha)=y^{k}\end{cases}
$$

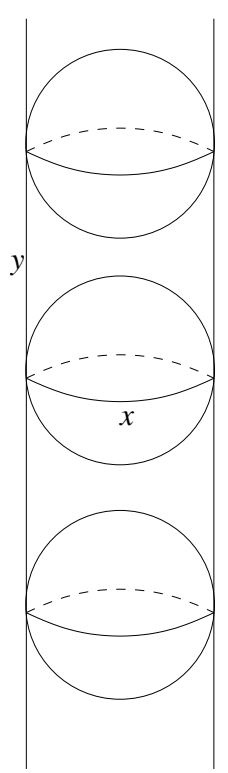

Fig. 3 
Theorem 5.14. Let $K$ be a finite 3-complex. There is no map $f: K \rightarrow$ $S^{1} \times \mathbb{P}^{2}$ which is strongly surjective if one of the following conditions holds:

(i) $H^{3}(K ; \widetilde{\mathbb{Z}})=0$,

(ii) $H^{3}(K ; \widetilde{\mathbb{Z}})$ is finite and $H^{3}\left(K ; \mathbb{Z}\left[\mathbb{Z} \times \mathbb{Z}_{2}\right]\right)$ is torsion free.

Proof. (i) Since $S^{1} \times \mathbb{P}^{2}$ has two cells in dimension $2, \widetilde{f}_{*}^{2}\left(\widetilde{e}_{i}^{2}\right)=\left(c_{i}, d_{i}\right)$ for $i=1, \ldots, n$, and $c_{i}, d_{i} \in \mathbb{Z}\left[\mathbb{Z} \times \mathbb{Z}_{2}\right]$. By Theorem 5.6 the systems $P x=$ $k(x+1)$ and $P x=k(y-1)$ have as solutions the vectors $c=\left[c_{1}, \ldots, c_{n}\right]^{t}$ and $d=\left[d_{1}, \ldots, d_{n}\right]^{t}$, respectively. From the proof of Lemma 3.3, the assumption $H^{3}(K ; \widetilde{\mathbb{Z}})=0$ means that the $m \times m$ minors $d_{1}, \ldots, d_{r}$ of $P$ have the property that the integers $\widetilde{\varepsilon}\left(d_{1}\right), \ldots, \widetilde{\varepsilon}\left(d_{r}\right)$ are relatively prime. From Lemma 2.4, there exist integers $p_{1}, \ldots, p_{r}$ and elements $q_{1}, q_{2} \in \mathbb{Z}\left[\mathbb{Z} \times \mathbb{Z}_{2}\right]$ such that

$$
p_{1} d_{1}+\cdots+p_{r} d_{r}-q_{1}(x+1)-q_{2}(y-1)=1 .
$$

This means that a maximal ideal $\mathfrak{m}$ of $\mathbb{Z}\left[\mathbb{Z} \times \mathbb{Z}_{2}\right]$ cannot contain the set $\left\{d_{1}, \ldots, d_{r}, x+1, y-1\right\}$. From [HG, Proposition 1], the system $P x=k$ has a solution.

(ii) Since $H^{3}(K ; \widetilde{\mathbb{Z}})$ is finite, some minors $d_{i}$ have $\widetilde{\varepsilon}\left(d_{i}\right) \neq 0$. Similar to the previous proof, we can show that $P x=k \widetilde{\varepsilon}\left(d_{i}\right)$ has a solution.

REMARK 5.15. By Lemma 3.3, the hypothesis that $H^{3}(K ; \widetilde{\mathbb{Z}})$ is finite in (ii) of the above theorem may be replaced by $H^{3}(K ; \mathbb{Z})$ being finite of odd cardinality.

Next we will consider the torus of dimension three, $T^{3}=S^{1} \times S^{1} \times S^{1}$. The three-dimensional torus is obtained from the unit cube by identifying the opposite faces (see Figure 4). A $C W$-decomposition of $T$ is obtained in the following way: Its 1-skeleton $K^{1}=\bigvee_{i}^{3} S_{i}^{1}$ is the bouquet of three circles, and the 2-skeleton $K^{2}$ has three 2-cells attached via the maps

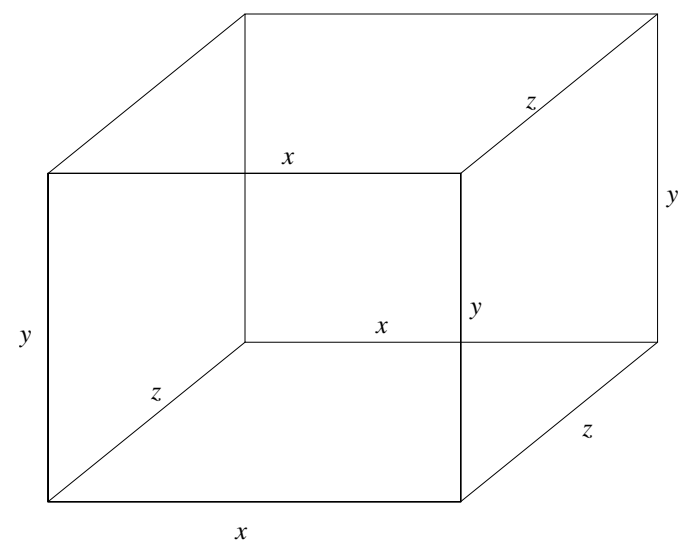

Fig. 4 
$x y x^{-1} y^{-1}, z x z^{-1} x^{-1}, y z y^{-1} z^{-1}$. Notice that $K^{2}$ is the union of three tori $T_{[x, y]}, T_{[z, x]}$, and $T_{[y, z]}$, and its universal cover is the subspace of $\mathbb{R}^{3}$ given by the union $\widetilde{K}^{2}=(\mathbb{R} \times \mathbb{R} \times \mathbb{Z}) \cup(\mathbb{R} \times \mathbb{Z} \times \mathbb{R}) \cup(\mathbb{Z} \times \mathbb{R} \times \mathbb{R})$. The covering map $p: \widetilde{K}^{2} \rightarrow K^{2}$ is the usual map corresponding to the 2-dimensional torus, when restricted to each plane. To get $T^{3}$, it is enough to attach a 3 -cell to $K^{2}$ by the quotient map restricted to the boundary of a cube, where the boundary is inside $\widetilde{K}^{2}$.

The boundary operator

$$
\widetilde{\partial}_{T^{3}}: \mathbb{Z}\left[x, y, z, x^{-1}, y^{-1}, z^{-1}\right] \rightarrow \bigoplus_{i=1}^{3} \mathbb{Z}\left[x, y, z, x^{-1}, y^{-1}, z^{-1}\right]
$$

is given by $\widetilde{\partial}_{T^{3}}(b)=b(x-1, y-1, z-1)$. The elements $x-1, y-1, z-1$ are all irreducible in $\mathbb{Z}[x, y, z]$, so the ideals generated by them are prime. It follows they are also prime ideals of $\mathbb{Z}\left[x, y, x, x^{-1}, y^{-1}, z^{-1}\right]$.

Theorem 5.16. Let $K$ be a finite 3-complex. There is no map $f: K \rightarrow$ $T^{3}$ which is strongly surjective if one of the following conditions holds:

(i) $H^{3}(K ; \mathbb{Z})=0$,

(ii) $H^{3}(K ; \mathbb{Z})$ is finite and $H^{3}(K ; \mathbb{Z}[\mathbb{Z} \times \mathbb{Z} \times \mathbb{Z}])$ torsion free,

(iii) $H^{3}(K ; \mathbb{Z})$ is finite and $K$ square.

Proof. (i) Since $T^{3}$ has three cells of dimension $2, \widetilde{f}_{*}^{2}\left(\widetilde{e}_{i}^{2}\right)=\left(b_{i}, c_{i}, d_{i}\right)$ for $i=1, \ldots, n$ and $b_{i}, c_{i}, d_{i} \in \mathbb{Z}[\mathbb{Z} \times \mathbb{Z} \times \mathbb{Z}]$. From Theorem 5.6, the systems $P x=k(x-1), P x=k(y-1), P x=k(z-1)$ have solutions $b=\left[b_{1}, \ldots, b_{n}\right]^{t}$, $c=\left[c_{1}, \ldots, c_{n}\right]^{t}, d=\left[d_{1}, \ldots, d_{n}\right]^{t}$, respectively. As before, the hypothesis $H^{3}(K ; \mathbb{Z})=0$ implies that the minors $d_{1}, \ldots, d_{r}$ have augmentations relatively prime. From Lemma 2.3, it follows that the system $P x=k$ has a solution.

(ii) The proof is analogous to the proof of the preceding theorem.

(iii) Since $P$ is a square matrix, we can apply Cramer's rule for the system $P b=k(x-1)$. Therefore $\operatorname{det}(P) b_{i}$

$$
\begin{aligned}
=\operatorname{det}\left[\begin{array}{cccccc}
f_{\#}\left(\widetilde{a}_{11}\right) & \ldots & f_{\#}\left(\widetilde{a}_{1, i-1}\right) & k_{1}(x-1) & f_{\#}\left(\widetilde{a}_{1, i+1}\right) & f_{\#}\left(\widetilde{a}_{n 1}\right) \\
\vdots & & \vdots & & \vdots \\
f_{\#}\left(\widetilde{a}_{1 n}\right) & \ldots & f_{\#}\left(\widetilde{a}_{n, i-1}\right) & k_{n}(x-1) & f_{\#}\left(\widetilde{a}_{n, i+1}\right) & f_{\#}\left(\widetilde{a}_{n n}\right)
\end{array}\right] \\
=(x-1) \operatorname{det}\left[\begin{array}{cccccc}
f_{\#}\left(\widetilde{a}_{11}\right) & \ldots & f_{\#}\left(\widetilde{a}_{1, i-1}\right) & k_{1} & f_{\#}\left(\widetilde{a}_{1, i+1}\right) & f_{\#}\left(\widetilde{a}_{n 1}\right) \\
& \vdots & & \vdots & & \vdots \\
f_{\#}\left(\widetilde{a}_{1 n}\right) & \ldots & f_{\#}\left(\widetilde{a}_{n, i-1}\right) & k_{n} & f_{\#}\left(\widetilde{a}_{n, i+1}\right) & f_{\#}\left(\widetilde{a}_{n n}\right)
\end{array}\right] .
\end{aligned}
$$


This means that $\operatorname{det}(P) b_{i}$ belongs to the ideal generated by $x-1$. The fact that $H^{3}(K ; \mathbb{Z})$ is finite implies that the augmentation of $\operatorname{det}(P)$ is not zero. Since every element of $(x-1)$ has augmentation zero, $\operatorname{det}(P) \notin(x-1)$, hence $b_{i} \in(x-1)$, therefore $(x-1)$ is prime. It follows that $b=\left[q_{1}, \ldots, q_{n}\right]^{t}(x-1)$, where $q_{i} \in \mathbb{Z}[\mathbb{Z} \times \mathbb{Z} \times \mathbb{Z}]$. Then $P q=k$ for $q=\left[q_{1}, \ldots, q_{n}\right]^{t}$.

\section{References}

[A] C. Aniz, Raízes de funções de um complexo em uma variedade, Doctoral Thesis, Universidade de São Paulo, Instituto de Ciências Matemáticas e de Computação, São Carlos, 2002.

[BG1] L. D. Borsari and D. L. Gonçalves, A van Kampen type theorem for coincidences, Topology Appl. 101 (2000), 149-160.

[BG2] -, 一, Obstruction theory and minimal number of coincidences for maps from a complex into a manifold, Topol. Methods Nonlinear Anal. 21 (2003), 115-130.

[BR1] R. Brooks, On the sharpness of the $\Delta_{2}$ and $\Delta_{1}$ Nielsen numbers, J. Reine Angew. Math. 259 (1973), 101-108.

[BR2] -, Coincidences, roots and fixed points, Doctoral Dissertation, Univ. of California, Los Angeles, 1967.

[BR3] -, On removing coincidences of two maps when only one, rather than both, of them may be deformed by a homotopy, Pacific J. Math. 39 (1971), 45-52.

[G] D. L. Gonçalves, Coincidence theory for maps from a complex to a manifold, Topology Appl. 92 (1999), 63-77.

[HG] J. A. Hermida and T. Sánchez-Giralda, Linear equations over commutative rings and determinantal ideals, J. Algebra 99 (1986), 72-79.

[HMS] C. Hog-Angeloni, W. Metzler and A. J. Sieradski, Two-Dimensional Homotopy and Combinatorial Group Theory, London Math. Soc. Lecture Note Ser. 197, Cambridge Univ. Press, 1993.

[H] S. T. Hu, Homotopy Theory, Academic Press, New York and London, 1959.

[TK] T. H. Kiang, The Theory of Fixed Point Classes, Springer, Berlin, and Science Press, Beijing, 1989.

[M] J. R. Munkres, Elements of Algebraic Topology, Benjamin/Cummings, 1984.

[WG] G. W. Whitehead, Elements of Homotopy Theory, Springer, 1978.

Departamento de Matemática

Universidade Federal de Mato Grosso do Sul - UFMS

Caixa Postal 549

79070-900/Unidade V/Campo Grande, MS, Brasil

E-mail: caniz@dmt.ufms.br 